\title{
Inhibition of Dual-specificity phosphatase 14 (DUSP14) by Ethyl-3,4-dephostatin
}

\author{
Huiyun Seo and Sayeon Cho* \\ College of Pharmacy, Chung-Ang University, Seoul 156-756, Korea. *E-mail: sycho@cau.ac.kr \\ Received April 14, 2011, Accepted April 19, 2011
}

Key Words : DUSP14, Ethyl-3,4-dephostatin, PTP inhibitor

Most cellular functions derived from signal transduction pathways require protein phosphorylation. ${ }^{1}$ Protein phosphorylation is processed by opposing activities of protein kinases and protein phosphatases. Dual-specificity phosphatases (DUSPs) are a subclass of protein tyrosine phosphatases (PTP) families that comprises 107 genes in human genome and can dephosphorylate both phosphotyrosine and phosphoserine/phosphothreonine residues on substrates. $^{2}$ Some DUSPs have been reported as key regulators for inactivating mitogen-activated protein kinases (MAP kinase). ${ }^{3}$ The three major subfamilies of MAPK in mammalian cells are p38, extracellular signal regulated kinase (ERK), c-Jun N-terminal kinase (JNK). ${ }^{4}$

Most PTPs are related to various cellular biological processes and thus modulation of their enzymatic activities is important in regulating disease susceptibility. ${ }^{5}$ Therefore, specific chemical compounds that regulate the activity of PTPs may have potent therapeutic approaches for diseases.

Ethyl-3,4-dephostatin is known to inhibit PTP-1B and Src homology-2-containing protein tyrosine phosphatase-1 (SHP-1) selectively (Fig. 1). ${ }^{6,7}$ Since ethyl-3,4-dephostatin might inhibit other phosphatases, we performed in vitro phosphatase assays with recombinant PTPs in the presence of ethyl-3,4-dephostatin. DUSP14 was one of a few PTPs found to be strongly inhibited by ethyl-3,4-dephostatin.

When DUSP14 was treated with increasing concentrations of ethyl-3,4-dephostatin, its DUSP14 activity was decreased by ethyl-3,4-dephostatin in a dose-dependent manner. When an inhibition curve was plotted for DUSP14, the half maximal inhibitory concentration $\left(\mathrm{IC}_{50}\right)$ was about $9.7 \pm$ $0.02 \mu \mathrm{M}$ (Fig. 2(a)). $K_{m}$ value was determined to $14.46 \pm$ $4.05 \mu \mathrm{M}$. We then performed kinetic studies based on the Michaelis-Menten equation to examine the DUSP14 inhibitory mechanism by ethyl-3,4-dephostatin. Analysis of the mode of inhibition indicated a competitive inhibition with $K_{i}$ of $7.91 \mu \mathrm{M}$ (Fig. 2(b)), suggesting that ethyl-3,4dephostatin inhibits the activity of DUSP14 through binding to the catalytic site.

It has been suggested that DUSP14 dephosphorylates

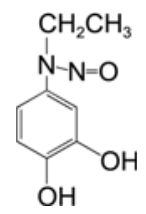

Figure 1. Chemical structure of ethyl-3,4-dephostatin.
phospho-ERK, phospho-JNK, and phospho-p38, suggesting that DUSP14 behaves as a general MAPK phosphatase. ${ }^{8}$ To examine that ethyl-3,4-dephostatin could function as a DUSP14 inhibitor and thus activate JNK in cells, we measured the in vivo phosphorylation level of JNK. HEK 293 cells were transfected with FLAG-DUSP14 expression plasmid, pretreated with or without ethyl-3,4-dephostatin for $3 \mathrm{~h}$, and then stimulated with $\mathrm{H}_{2} \mathrm{O}_{2}$ to phosphorylate JNK. Levels of phospho-JNK were determined with immunoblotting analysis. Ethyl-3,4-dephostatin activates JNK kinase activity by inhibiting DUSP14 in vivo (Fig. 3). These results suggest that ethyl-3,4-dephostatin recovers DUSP14-suppressed JNK activity by inhibiting DUSP14.

In conclusion, we found that ethyl-3,4-dephostatin acts as an effective inhibitor of DUSP14. DUSP14 has been reported to a negative regulator of CD28 signaling through dephosphorylation of MAPKs in T cells. ${ }^{8}$ We found that ethyl-3,4-dephostatin inhibits DUSP14 activity and thus recovers JNK activity. Therefore, our study suggests that

(a)

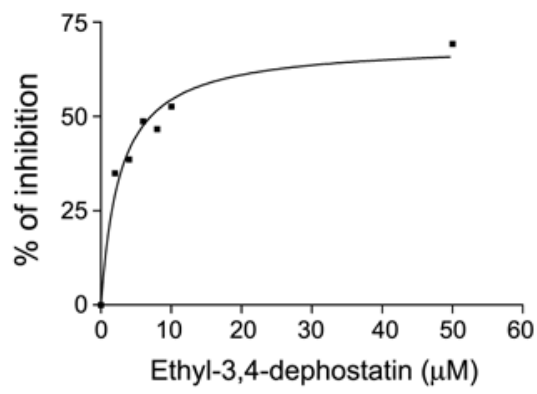

(b)

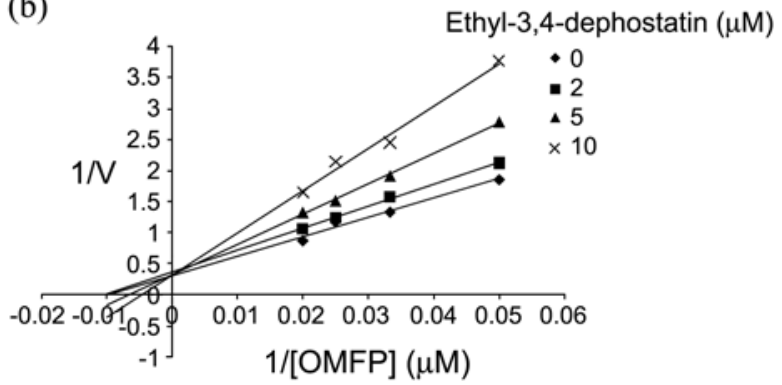

Figure 2. Inhibitory effect of ethyl-3,4-dephostatin on DUSP14 and Kinetic analysis of DUSP14 inhibition by ethyl-3,4-dephostatin. (a) DUSP14 were incubated with various concentrations of ethyl-3,4-dephostatin at $37{ }^{\circ} \mathrm{C}$ for $30 \mathrm{~min}$. Fluorescence emission from the product was measured with a multiwell plate reader as described in Experimental section. (b) Lineweaver-Burk plots of DUSP14 were generated from the reciprocal data. 


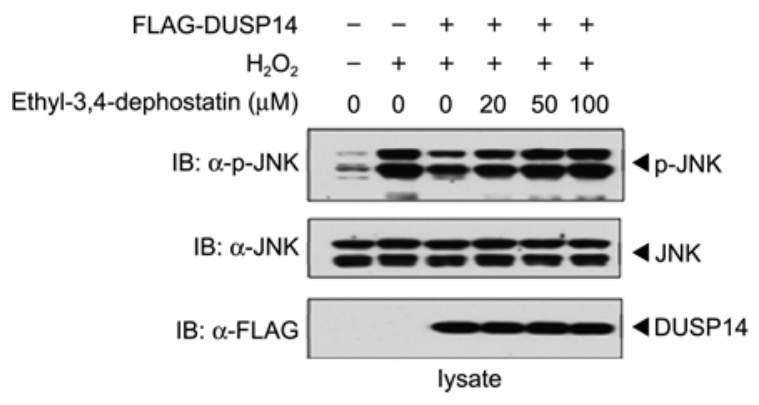

Figure 3. Ethyl-3,4-dephostatin inhibits DUSP14-dependent JNK activation. Transfected HEK 293 cells were pre-treated with various concentrations of ethyl-3,4-dephostatin $(0,20,50,100 \mu \mathrm{M})$ for $3 \mathrm{~h}$ and then stimulated with $\mathrm{H}_{2} \mathrm{O}_{2}(1 \mathrm{mM}, 30 \mathrm{~min})$. Cell lysates were analyzed by immunoblotting with appropriate antibodies as described.

ethyl-3,4-dephostatin could be applied to the development of novel therapeutics for immune diseases related to DUSP14mediated T-cell inactivation.

\section{Experimental Section}

Antibodies. Anti phospho-JNK (specific for phosphoThr183 and phospho-Tyr185) was purchased from Cell Signaling Technology (Danvers, MA). Anti-JNK antibody was from Santa Cruz Biotechnology (Santa Cruz, CA).

Cell culture and Transfection. Human embryonic kidney (HEK) 293 cells were maintained at $37^{\circ} \mathrm{C}$ in Dulbecco's modified Eagle's medium (DMEM, Invitrogen, Carlsbad CA) supplemented with $10 \%$ fetal bovine serum (FBS, Invitrogen) and penicillin/streptomycin in the presence of $5 \% \mathrm{CO}_{2}$. For transient transfection, $1.4 \times 10^{6}$ cells were plated in each 60 $\mathrm{mm}$ cell culture plate, grown overnight, and transfected with DNA using LipofectAMINE (Invitrogen).

Plasmid Constructions. FLAG-tagged DUSP14 were constructed in pcDNA3.1 (Invitrogen). His-tagged DUSP14 was constructed in pET28a plasmid (Novagen, Darmstadt, Germany) for protein expression in Escherichia coli.

Purification of the six-His-tagged DUSP14. Purification of recombinant proteins was carried out as previously described. ${ }^{9}$

In vitro Phosphatase Assays and Kinetic Analysis. The activity of DUSP14 was measured using the substrate 3Omethylfluorescein phosphate (OMFP; Sigma) in a 96-well microtiter plate assay based on methods described previously. ${ }^{10}$ The ethyl-3,4-dephostatin (Sigma) and OMFP were solubilized in $\mathrm{H}_{2} \mathrm{O}$ and DMSO, respectively. All reactions were performed at a final concentration of $1 \%$ DMSO. The final incubation mixture $(150 \mu \mathrm{L})$ was optimized for enzyme activity and composed of $30 \mathrm{mM}$ Tris- $\mathrm{HCl}(\mathrm{pH}$ 7), $75 \mathrm{mM}$ $\mathrm{NaCl}, 1 \mathrm{mM}$ ethylenediaminetetraacetic acid (EDTA), 0.1 $\mathrm{mM}$ dithiothreitol (DTT), $0.33 \%$ bovine serum albumin (BSA) and $100 \mathrm{nM}$ of DUSP14. Reactions were initiated by addition of OMFP and incubated for $30 \mathrm{~min}$ at $37^{\circ} \mathrm{C}$. Fluorescence emission from product was measured with a multi-well plate reader (GENios Pro; excitation filter, 485 $\mathrm{nm}$; emission filter, $535 \mathrm{~nm}$ ). The reaction was linear over the time period of the experiment and was directly proportional to both enzyme and substrate concentration. Halfmaximal inhibition constant $\left(\mathrm{IC}_{50}\right)$ was defined as the concentration of an inhibitor that caused a $50 \%$ decrease in the DUSP14 activity. Half-maximal inhibition constants and best curve fit for Lineweaver-Burk plots were determined by using the curve fitting program Prism 3.0 (GraphPad Software). All experiments were performed in triplicate and were repeated at least three times.

Immunoblotting Analysis. Samples were run in SDS$10 \%$ polyacrylamide gels and transferred to nitrocellulose membrane. The membrane was blocked in 5\% nonfat skim milk and incubated with an appropriate antibody, followed by incubation with a secondary antibody conjugated to horseradish peroxidase. The immunoreactive bands were visualized using an ECL system (Pierce, Rockford, IL).

Inhibition Study. The inhibition constant $\left(K_{i}\right)$ to DUSP14 phosphatase for the inhibitor was determined by measuring the initial rates at several OMFP concentrations for each fixed concentration of the inhibitor. The data were fitted to the following equation to obtain the inhibition constant of reversible competitive inhibitors. The slopes obtained were replotted against the inhibitor concentrations. The $K_{i}$ value was obtained from the slopes of these plots. ${ }^{11}$

$$
1 / V=K_{m}\left(1+[\mathrm{I}] / K_{i}\right) V_{\max }[\mathrm{S}]+1 / V_{\max }
$$

Acknowledgments. This Research was supported by the Chung-Ang University Research Grants in 2010.

\section{References}

1. Hunter, T. Cell 2000, 100, 113-127.

2. Patterson, K. I.; Brummer, T.; O'Brien, P. M.; Daly, R. J. Biochem J. 2009, 418, 475-489.

3. Lewis, T. S.; Shapiro, P. S.; Ahn, N. G. Adv. Cancer Res. 1998, 74, 49-139.

4. Schaeffer, H. J.; Weber, M. J. Mol. Cell Biol. 1999, 19, $2435-$ 2444.

5. Fischer, E. H.; Charbonneau, H.; Tonks, N. K. Science 1991, 253, 401-406.

6. Suzuki, T.; Hiroki, A.; Watanabe, T.; Yamashita, T.; Takei, I.; Umezawa, K. J. Biol. Chem. 2001, 276, 27511-27518.

7. Watanabe, T.; Suzuki, T.; Umezawa, Y.; Takeuchi, T.; Otsuka, M.; Umezawa, K. Tetrahedron 2000, 56, 741-752.

8. Marti, F.; Krause, A.; Post, N. H.; Lyddane, C.; Dupont, B.; Sadelain, M.; King, P. D. J. Immunol. 2001, 166, 197-206.

9. Song, M.; Cho, S. Bull. Korean Chem. Soc. 2009, 30, 1190-1192.

10. Tierno, M. B.; Johnston, P. A.; Foster, C.; Skoko, J. J.; Shinde, S N.; Shun, T. Y.; Lazo, J. S. Nat. Protoc. 2007, 2, 1134-1144.

11. Shi, Z.; Tabassum, S.; Jiang, W.; Zhang, J.; Mathur, S.; Wu, J.; Shi, Y. Chembiochem. 2007, 8, 2092-2099. 